Pneumologe 2018 $\cdot 15: 297-298$

https://doi.org/10.1007/s10405-018-0207-5

(c) Springer Medizin Verlag GmbH, ein Teil von Springer Nature 2018

CrossMark

\section{Claus Kroegel' • Jens Schreiber ${ }^{2}$}

${ }^{1}$ Abt. Pneumologie \& Allergologie/Immunologie, Medizinische Klinik I, Universitätsklinikum Jena, Jena, Deutschland

${ }^{2}$ Universitätsklinik für Pneumologie, Otto-von-Guericke-Universität Magdeburg, Magdeburg, Deutschland

\title{
Der eosinophile Granulozyt - vom Ursprung bis zur therapeutischen Zielzelle
}

Beim eosinophilen Granulozyten handelt es sich um eine enigmatische Zelle unseres Immunsystems. Sie fällt nicht nur durch die leuchtend orange Farbe nach Eosinfärbung auf, sondern sie besitzt auch die größten zellulären Granula innerhalb aller Immunzellen. Schließlich hat ihre Entdeckung ebenso wie die Zuordnung einer immunologischen Funktion einen langen kurvenreichen Weg genommen.

Der Eosinophile ist 1846 T. WhartonJones vermutlich erstmals als grobkörnige Struktur aufgefallen [7]. Er interpretierte dies als „grobkörniges Stadium“ bei der Entwicklung von Granulozyten in tierischem und menschlichem Blut. Einige Jahre später erkannte Max Schultze die sehr grobkernigen Zellen nicht nur als Entwicklungsstufe, sondern als Charakteristikum einer Zelle, die sich durch ihre körnige Struktur von anderen unterscheidet [6]. Als eigenständiger Zelltyp wurde sie 1879 von Paul Ehrlich charakterisiert [1], der sich mit der Differenzierung von Zellen anhand ihrer Färbeeigenschaften beschäftigte. Ihre intensive Affinität für Eosin und die grobkernige Zelle bildeten die Grundlage für die heute noch aktuelle Bezeichnung „eosinophiler Granulozyt“.

\section{》) Der eosinophile Granulozyt gilt heute als multifunktionale Zelle}

Nach ihrer Entdeckung wurden eosinophile Granulozyten noch im späten 19. Jahrhundert bei vielen Krankheiten beschrieben. Die Rolle der Zelle blieb jedoch unbekannt und sie wurde zum Objekt zahlreicher Spekulationen [4]. Vor Beginn des 19. Jahrhunderts wurden sie mit parasitären Infestationen in Verbindung gebracht und eine Rolle bei der Abwehr von Parasiten angenommen [3]. Noch in den 60er und 70er Jahren des letzten Jahrhunderts führte ihr verzögertes Auftreten im Rahmen von Entzündungsreaktionen zur Auffassung, dass es sich um am Heilungsprozess beteiligte Zellen handelt. Sie wurde auch als „Morgenröte der Entzündung“ angesehen [2]. Erst mit der weitergehenden Charakterisierung ihrer Zellbestandteile und dem Verständnis von der Wirkung der vom Eosinophilen freigesetzten Mediatoren, insbesondere der basischen Proteine in den sekundären Granula, die für die auffallend grobe Granulation der Zelle verantwortlich sind, sowie der $\mathrm{Zu}$ sammenhang der Eosinophilenzahl im Blut und im Gewebe mit der Krankheitsintensität führten im Lauf der 1980er Jahre allmählich zur Auffassung, dass es sich beim eosinophilen Granulozyten um eine proentzündliche Effektorzelle handelt [2]. Der Einsatz moderner Methoden, einschließlich von „Knockout“Modellen, hat dazu beigetragen, die Funktion der Zelle zu charakterisieren [4]. Heute gilt der eosinophile Granulozyt als eine multifunktionale Zelle, die einerseits eine entzündliche Reaktion bis hin zum nekrotischen Gewebeuntergang induziert. Andererseits hat er modulierende Funktionen im Rahmen des immunologischen Netzwerks und ist in bestimmten Situationen an der Verteidigung des Wirts bei parasitären In- 
festationen beteiligt [5]. Der Eosinophile ist das Charakteristikum einer Gruppe bronchopulmonaler Erkrankungen, die als „eosinophile Lungenerkrankungen“ zusammengefasst werden. Diesem Spektrum pneumologischer Erkrankungen widmet sich das vorliegende Themenheft.

\section{》) Der eosinophile Granu- lozyt ist für verschiedene Krankheitsbilder verantwortlich}

Im klinischen Alltag bildet die Eosinophilie meist eine differenzialdiagnostische Herausforderung. Dieser Aspekt wird von J. Schreiber im Detail besprochen. Die potenziellen Ursachen eosinophiler Erkrankungen reichen von allergischen bzw. immunologischen, infektiösen, neoplastischen und medikamenteninduzierten Erkrankungen bis hin zu idiopathischen Krankheitsbildern. Ein Beispiel für eine Eosinophilie unbekannter Ursache sind die eosinophilen Pneumonien und das hypereosinophile Syndrom (HES), die von J. Wälscher und M. Kreuter zusammengefasst werden. Auf die Eosinophilie infektiöser Ursache vor allem durch Parasiten geht die Arbeit von Moeser et al. ein. Bei diesen Systemerkrankungen ist die Lunge oft betroffen. Eine Erkrankung mit immunologischem und allergischem Hintergrund bildet die eosinophile Granulomatose mit Polyangiitis (EGPA, früher: Churg-StraussSyndrom), die von C. Kroegel et al. dargestellt wird. $\mathrm{Zu}$ den eosinophilenassoziierten Erkrankungen allergischer bzw. immunologischer Ursache gehören auch das Asthma bronchiale und die exogen-allergische Alveolitis, die von Lommatzsch und Mitarbeitern zusammengefasst werden. Das verbindende Element bei diesen Erkrankungen ist der eosinophile Granulozyt. Er ist mittels seiner zellulären Effektorfunktionen wesentlich für die pulmonalen und extrapulmonalen Gewebe- bzw. Organläsionen und damit für die klinische Präsentation der verschiedenen Krankheitsbilder verantwortlich. Daher wird eine kurze Übersicht zur Zellbiologie des eosinophilen Granulozyten von C. Kroegel den eosinophilenassoziierten Erkrankungen vorangestellt.
Die Entwicklung neuer selektiver Therapeutika, die über die Neutralisation des Interleukin-5 (IL-5) oder über die Blockade des IL-5-Rezeptors eosinophile Granulozyten eliminieren können, bietet nun die Voraussetzung, deren Funktion noch besser zu verstehen und deren Rolle im Rahmen bestimmter Erkrankungen zu definieren. Aus klinischer Sicht ergibt sich zugleich die Möglichkeit, hochselektiv eosinophilenassoziierte Erkrankungen zu behandeln. Die betrifft nicht nur das Asthma bronchiale, sondern ebenso immunologische Erkrankungen sowie hämatologische und infektiöse Krankheiten.

Mit der Verfügbarkeit von Mepolizumab, Reslizumab und Benralizumab drängt sich daher die Frage auf, welche Konsequenzen sich durch diese neuen Therapieoptionen für pneumologische, allergologische, hämatologische und infektiöse Erkrankungen ergeben. Aus diesem Grund wurde das jetzt vorliegende Themenheft konzipiert. Es soll einerseits die Bedeutung des eosinophilen Granulozyten als pathogenetische Effektorzelle, aber auch als Biomarker und als therapeutische Zielzelle bei verschiedenen Erkrankungen darstellen und die sich daraus ableitenden diagnostischen sowie therapeutischen Konsequenzen diskutieren.

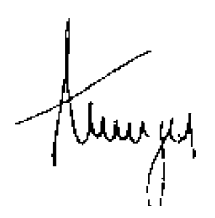

Prof. Dr. Claus Kroegel

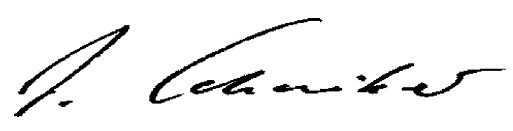

Prof. Dr. Jens Schreiber

\section{Korrespondenzadresse}

\section{Prof. Dr. C. Kroegel}

Abt. Pneumologie \& Allergologie/

Immunologie, Medizinische Klinik I,

Universitätsklinikum Jena

Am Klinikum 1, 07740 Jena, Deutschland

Claus.Kroegel@med.uni-jena.de

Interessenkonflikt. C. Kroegel und J.Schreiber geben an, dass kein Interessenkonflikt besteht.

\section{Literatur}

1. Ehrlich $P$ (1879) Ueber die spezifischen Granulationen des Blutes. Archiv für Anatomie und Physiologie. Physiologische Abteilung, S 571-579

2. Gleich GJ (2013) Historical overview and perspective on the role of the eosinophil in health and disease. In: Lee JJ, Rosenburg HF (Hrsg) Eosinophils in Health and Disease. Academic Press, Waltham, $\mathrm{S} 1-12$

3. Kay A (2014) The early history of the eosinophil. Clin Exper Allergy 45:575-582

4. Radonjic-Hösli S, Simon H-U (2014) Eosinophils. In: Bergmann K-C, Ring J (Hrsg) History of Allergy. Chem Immunol Allergy, Bd. 100. Karger, Basel, S 193-204

5. Rothenberg ME, Hogan SP (2006) The Eosinophil. Annu Rev Immunol 24:147-174

6. Schultze MJS (1865) Ein heizbarer Objecttisch und seine Verwendung bei Untersuchungen des Blutes. Archiv für mikroskopische Anatomie, I. Bd. S1-42. https://link.springer.com/content/pdf/10. 1007/BF02961404.pdf

7. Wharton Jones $T$ (1846) The blood corpuscle considered in it's different phases of development in the animal series. Memoir 1, vertebrata. Philos Trans RSoc Lond 1:63-87 